\title{
Falls Resulting in Spinal Cord Injury: Patterns and Outcomes in an Older Population
}

S. I. Weingarden, MD, ${ }^{1}$ P. M. Graham, RN, MS, CRRN ${ }^{2}$

${ }^{1}$ Project Director, ${ }^{2}$ System Co-ordinator, Southeastern Michigan Spinal Cord Injury System, Rehabilitation Institute, 261 Mack Boulevard, Detroit, Michigan 48201, USA

\section{Summary}

Falls are the major cause of spinal cord injuries in older people. The pattern of injury seen most frequently is that of a central cord syndrome due to cervical hyperextension. The medical records of 58 patients over 50 years of age who sustained a spinal cord injury in a fall were reviewed for circumstances of onset, length of stay and outcomes of rehabilitation.

The study identified elderly single or widowed men, and those who use alcohol, as high risk groups.

Keywords: Spinal cord injuries; Falls; Central cord syndrome.

Spinal cord injury (SCI) is considered a 'disease' of the young. The elderly, however, are not immune; although the aetiologies and neurological patterns differ from those of young patients. Trauma from motor vehicle or diving accidents, and injuries as a result of violence also occur among an older population; but falls account for the majority of the injuries. The pattern seen most frequently is central cord syndrome (CCS); 'a lesion almost exclusively in the cervical region, involving the central grey matter and the more medial white matter producing greater weakness in the upper limbs than in the lower limbs and sacral sensory sparing' (ASIA, 1989). This type of injury is most often caused by cervical hyperextension. The purpose of this paper is to review results of a study of 58 patients over 50 years of age who sustained a SCI secondary to a fall, and to examine those pre-event factors that may have contributed to the accident.

\section{Literature review}

Shrosbree reviewed a series of 99 patients with acute central cord lesions (Shrosbree, 1977). He reported that 30 were over the age of 50, and $16(53 \%)$ of 
these 30 were injured in falls. It was also noted that $76 \%$ or 23 of the injuries in those over 50 were due to cervical hyperextension.

Hardy reviewed a series of 141 patients with cervical injuries that occurred without skeletal damage (Hardy, 1977). Seventy per cent were over the age of 50 , with falls stated as the most common cause. He noted that alcohol may have played a role in some of the injuries. Clinically, the pattern seen most frequently was that of CCS with paralysis 'most marked in the hands and less so in the legs'. The Brown-Sequard pattern was also noted in that 'one side was almost invariably worse than that of the other'.

In 1985, Foo et al. reviewed medical records of patients admitted to a Veterans Administration Hospital with ankylosing spondylitis and who also sustained cervial spinal cord injuries (Foo et al., 1985). Six of the 7 were injured in a fall: 3 fell while walking; 1 fell from a bed; 1 fell down a flight of stairs; and 1 fell from a height of 20 feet. In 5 of the 7 cases, alcohol use preceded the accident. Ankylosing spondylitis apparently put the patient at high risk for SCI from what would otherwise have been an inconsequential fall. Safety precautions such as hand rails, night lights, and avoidance of intoxicants were advised.

In a later study (Foo, 1986) of 44 SCI patients with cervical spondylosis, 22 $(50 \%)$ were injured from a minor fall, and $12\left(27^{\circ}\right.$ o $)$ of these gave a history of alcohol use. There was no breakdown pertaining to the cause of the injury for this subgroup although CCS was noted to be present.

Central cord syndrome secondary to an inconsequential fall, is frequently seen in people over 50 years of age and alcohol use may be a contributing factor. Alcohol has been implicated in other studies of falls. A hospital review by the Washington State Department of Social \& Health Services (1977) observed that in 1740 cases of falls, alcohol could be implicated in $10^{\circ} \%$ Macdonald reported that alcohol combined with subclinical proprioceptive impairment, poor eyesight, or cardiac disease, increased the risk of falls in the elderly (Macdonald, 1985).

Disease processes alone or in combination with the medications used to treat these problems also have been implicated in causing falls. Lipsitz observed that abnormalities in blood pressure homeostasis contribute to the risk of falling (Lipsitz, 1985). Any of these factors in isolation or combination may add to the risk of falls in the elderly. A final consideration noted (Gulino and Kadin, 1986) is that the use of alcohol in this age group is usually associated with loneliness and social isolation. Elderly widowers were the most likely group to abuse alcohol $(10 \cdot 5 \%)$.

\section{Method}

Medical records of all SCI patients over the age of 50 who were admitted to the Southeastern Michigan Spinal Cord Injury System between 1983 and 1988 were reviewed. A total of 75 patients over 50 years of age were admitted during this period: $58(77 \%)$ of these were injured secondary to a fall.

All subjects signed informed consents for the abstraction of their medical records and other data collected at time of entry into the system. Records were obtained from emergency room admission, through the intensive care phase 
Table I Sex and race, by age

\begin{tabular}{lcccccc}
\hline Age & $\begin{array}{c}\text { Black } \\
\text { male }\end{array}$ & $\begin{array}{c}\text { White } \\
\text { male }\end{array}$ & $\begin{array}{c}\text { Black } \\
\text { female }\end{array}$ & $\begin{array}{c}\text { White } \\
\text { female }\end{array}$ & Total & \% \\
\hline $50-55$ & 10 & 1 & 0 & 0 & 11 & 19 \\
$56-60$ & 8 & 7 & 1 & 0 & 16 & 28 \\
$61-65$ & 3 & 2 & 2 & 1 & 8 & 13 \\
$66-75$ & 4 & 3 & 3 & 1 & 11 & 19 \\
$76+$ & 2 & 6 & 0 & 4 & 12 & 21 \\
Total & 27 & 19 & 6 & 6 & 58 & 100 \\
\hline
\end{tabular}

Table II Marital status by sex

\begin{tabular}{lcccc}
\hline & Male & Female & Total & $\%$ \\
\hline Married & 20 & 0 & 20 & 35 \\
Single & 10 & 2 & 12 & 21 \\
Sep/divorced & 12 & 1 & 13 & 22 \\
Widowed & 4 & 9 & 13 & 22 \\
Total & 46 & 12 & 58 & 100 \\
\hline
\end{tabular}

and the rehabilitation hospital stay. Records were assigned numbers to protect patient confidentiality. Variables examined included age, sex, race, type of fall, use of alcohol, neurological classification of the spinal cord injury, marital status, pre-existing medical conditions, length of stay and destination at time of discharge.

\section{Results}

The racial distribution pattern of the 58 patients $(57 \%$ black and $43 \%$ white) reflects that of the total population served by this Model SCI System. The sex ratio, however, is quite skewed $\left(79^{\circ}\right.$, male and $21 \%$ female) (Table I). The 12 females were either widowed, divorced, or single as compared to $57 \%$ of the males (Table II). The mean age for the total group was $65 \cdot 3$ years.

\section{Nature of injury}

The circumstances of the fall causing the injury was classified into 1 of 3 categories: falling down stairs (23 cases); falling from a height such as a porch or balcony (19 cases); and falling on a level surface (16 cases). The latter was preceded by a loss of consciousness in 10 cases. The predominant patterns of injury were CCS (37); paraplegia (15); and complete quadriplegia (6). The Brown-Sequard Syndrome was seen in varying degrees in the group with CCS. Two of the 37 patients with CCS, however, had a predominant Brown-Sequard pattern. The central cord syndrome occurred in 11 of the 16 cases of level surface falls. Seventeen of the 23 patients who fell down stairs and 9 of the 19 who fell from a height such as a porch or balcony developed CCS. The level of injury was mainly C5 for those with CCS and thoracic for the patients with paraplegia.

Alcohol use was seen in a large percentage of the patients. Thirty-one of the 58 patients $(53 \%)$ were noted to have consumed some alcohol prior to their fall. 
Table III Discharge destination

\begin{tabular}{|c|c|c|c|c|c|c|}
\hline & \multicolumn{2}{|c|}{ Male } & \multicolumn{2}{|c|}{ Female } & \multicolumn{2}{|c|}{ Total } \\
\hline & $\mathrm{n}$ & $\%$ & $\mathrm{n}$ & $0_{0}^{\circ}$ & $\mathrm{n}$ & $0_{0}$ \\
\hline Private residence & 25 & 54 & 5 & 42 & 30 & 52 \\
\hline Nursing home & 9 & 20 & 4 & 33 & 13 & 22 \\
\hline Deceased & 12 & 26 & 3 & 25 & 15 & 26 \\
\hline Total & 46 & 100 & 12 & 100 & 58 & 100 \\
\hline
\end{tabular}

Upon examining the rate of alcohol use in respect to type of injury, only 4 of the 15 patients with paraplegia, and 2 of the 6 patients with complete quadriplegia had consumed alcohol. In contrast, however, 25 of the 37 patients with $\operatorname{CCS}\left(68^{\circ}{ }_{\circ}\right)$ had consumed alcohol prior to their injury.

As expected, medical problems were seen throughout the entire series because of the ages of the patients. Heart disease, hypertension, and arthritis were the most frequently documented pre-existirig diseases.

Mean length of stay for the patients who survived and were transferred to the rehabilitation unit was 87 days. This consisted of a mean stay of 34 days in the spinal cord intensive care unit, and a 53-day rehabilitation hospital stay.

The mortality rate in this series was $26^{\circ}{ }_{0}$ (15 of 58). The rate, however, differed significantly by injury pattern. Of the 37 patients with CCS only 2 expired, but of the 15 paraplegics, 7 expired, and all 6 of the patients with complete quadriplegia expired.

Discharge destination differed for males and females. Only 9 of the 34 surviving males were transfered to nursing home facilities. In contrast, 5 of the 9 surviving females went to nursing homes (Table III). This may be a reflection of the premorbid difference in marital situations.

\section{Discussion}

Spinal cord injury is usually perceived to be a male disease. Risk taking, violence, and sports are usually activities of young males. The male predominance is, therefore, expected in that age group. In this series, however, aetiology was limited to falls among the middle aged and the elderly; therefore, a more equal mix seemed plausible. The 4:1 male/female ratio seen in this series requires an explanation.

Cervical spondylosis, appears to have a male predilection. Friedenberg et al. found that $60^{\circ}$. of the patients with degenerative disc disease of the cervical spine were male (Friedenberg et al., 1963). Nurick in a review of patients with cervical spondylitic myelopathy found a 2:1 male preponderance (Nurick, 1972). Satomi et al. and Roth et al. found a 4:1 male/female ratio in patients of all ages with CCS (Satomi et al., 1988; Roth et al., 1988). Cervical spondylosis, most likely with stenosis, appears to be a male disease as indicated in this study.

Risk factors were also evident. Only 20 of the 58 patients were married. This would suggest that living alone makes a person more vulnerable to falls and injuries. This is especially true among single or widowed females. Alcohol use also seems to play a major role since over half of the patients were injured after 
consuming alcohol in varying amounts. Alcohol use was even more prevalent in patients with CCS. In this group $68^{\circ}$ o had used alcohol. When these two factors are combined with the ever present medications used by patients over 50 , it is not surprising to find that falls lead to major injuries.

The final point requiring comment is that the mortality rate was high for the entire series $\left(26^{\circ}{ }_{0}\right)$. Although it varied with pattern of injury, it was much lower in the patients with CCS $\left(5 \%_{0}\right)$ and much higher among the patients with a complete cervical injury $\left(100^{\circ}{ }_{0}\right)$. High mortality rates have been noted for these patients in previous studies. It is likely that a fall resulting in an injury causing complete quadriplegia is more severe than one causing CCS. This may also explain the $47 \%$ mortality in the patients with paraplegia. Another possible explanation for the complete cervical injury mortality rate of $100 \%$ is that the mean age for this group was 78 years as compared to 64 years of age for the rest of the series.

\section{References}

Donovan WH 1989 Standards for Neurological Classification of Spinal Injury Patients. American Spinal Injury Association, pp. 14-17.

Foo D, SARKARTi M, MARCELINO V 1985 Cerivcal spinal cord injury complicating ankylosing spondylitis. Paraplegia 23:358-363.

Foo D 1986 Spinal cord injury in forty-four patients with cervical spondylosis. Paraplegia 24:301-306.

Friedenberg Z, MILler W 1983 Degenerative disc disease of the cervical spine. Fournal of Bone and foint Surgery 45:1171-1177.

Gulino C, Kadin M 1986 Aging and reactive alcoholism, Geriatric Nursing May, June: 148151.

HaRdy A 1977 Cervical spinal cord injury without bony injury. Paraplegia 14:296-305.

Lipsitz L 1985 Abnormalities in blood pressure homeostasis that contribute to falls in the elderly. Clinics in Geriatric Medicine 1:637-643.

MACDONALD J 1985 The role of drugs in falls in the elderly. Clinics in Geriatric Medicine 1:621635.

NuRICK S 1972 The pathogenesis of the spinal cord disorder associated with cervical spondylosis. Brain 95:87-100.

PoDOLSKy D 1985 The not so safe refuge: unintentional injuries in the home and at play. Alcohol World 9:4, 24-27.

Roth J., LAWLER M 1988 Functional outcome of central cord syndrome. Proceedings of the American Spinal Injury Association 14th meeting, abstracts.

SHROSBREE R 1977 Acute central cord syndrome - aetiology, age, incidence and relationship to the orthopaedic injury. Paraplegia 14:251-258.

Satomi K, Herabayaski K 1988 Clinical analysis of central cervical cord injury. Proceedings of the American Spinal Injury Association 14th meeting, abstracts.

STOver SL (ed) 1986 Spinal Cord Injury: The Facts and Figures, Distribution of Etiology within Age Groups 7:19. 